3. Калекин, А. А. Гидравлика и гидравлические машины [Текст] / А. А. Калекин// М.: «Мир», 2005. -512 с.

4. Идельчик, И. Е. Справочник по гидравлическим сопротивлениям [Текст] / И. Е. Идельчик// - М.: «Машиностроение», 1975. - 560 с.

Надійшла до редколегї 12.08.2019

УДК 532.516

\author{
Д. А. Редчиц*, С. В. Моисеенко**, И. Б. Чашина*, И. В. Выгоднер** \\ *Институт транспортных систем и технологий НАН Украины \\ **Херсонский национальный технический университет
}

\title{
ОБТЕКАНИЕ ЦИЛИНДРА И АЭРОДИНАМИЧЕСКОГО ПРОФИЛЯ С УЧЕТОМ ЛАМИНАРНО-ТУРБУЛЕНТНОГО ПЕРЕХОДА
}

\begin{abstract}
Проведено моделирование обтекания цилиндра и профиля NACA 4412 с использованием $\gamma$ Reө модели ламинарно-турбулентного перехода и без нее. Численное моделирование выполнено на базе осредненных по Рейнольдсу уравнений Навье-Стокса (URANS), с использованием дифференциальной однопараметрической модели турбулентности Spalart-Allmaras. Система исходных уравнений, записывалась относительно произвольной криволинейной системы координат. Согласование полей давления и скорости осуществлялось с помощью метода искусственной сжимаемости, модифицированного для расчета нестационарных задач. Интегрирование системы исходных уравнений проводилось численно с использованием метода контрольного объема. Для конвективных потоков использовалась противопоточная аппроксимация Rogers-Kwak, основанная на схеме Roе третьего порядка точности. В моделях турбулентности для аппроксимации конвективных слагаемых применялась схема TVD c ограничителем потоков ISNAS третьего порядка. Проведено сравнение результатов расчетов обтекания цилиндра с использованием модели ламинарно-турбулентного перехода и без нее. Показано, что при низких числах Рейнольдса, когда обтекание цилиндра носит ламинарный характер, а след турбулентный, использование модели турбулентности Spalart-Allmaras приводит к развитию турбулентного пограничного слоя на цилиндре и, как следствие, к изменению положения точки отрыва. Неправильное положение точки отрыва оказывает влияние на распределение давления в донной части цилиндра и на интегральные аэродинамические характеристики. Применение модели перехода позволяет адекватно воспроизвести ламинарный отрыв вблизи передней кромки профиля с последующим его присоединением. Применение одной только модели Spalart-Allmaras приводит к излишней генерации турбулентной вязкости. Показано, что применение $\gamma$-Re $\operatorname{Re}_{\theta}$ модели ламинарнотурбулентного перехода качественно и количественно улучшает результаты численного моделирования. Полученные результаты численного моделирования обтекания кругового цилиндра и аэродинамического профиля NACA 4412 хорошо согласуются с экспериментальными данными в широком диапазоне чисел Рейнольдса.

Ключевые слова: ламинарно-турбулентный переход, аэродинамический профиль, уравнения Навье-Стокса, модель турбулентности.
\end{abstract}

Проведено моделювання обтікання циліндра і профілю NACA 4412 з використанням $\gamma$-Reo моделі ламінарно-турбулентного переходу і без неї. Чисельне моделювання виконано на базі осереднених за Рейнольдсом рівнянь Нав'є-Стокса (URANS), з використанням диференціальної однопараметричної моделі турбулентності Spalart-Allmaras. Система вихідних рівнянь, записувалася щодо довільної криволінійної системи координат.

(с Редчиц Д.А., Моисеенко С.В., Чашина И. Б., Выгоднер И.А., 2019 
Узгодження полів тиску і швидкості здійснювалося за допомогою методу штучної стисливості, модифікованого для розрахунку нестаціонарних задач. Інтегрування системи вихідних рівнянь проводилося чисельно 3 використанням методу контрольного об'сму. Для конвективних потоків використовувалася протипотокова апроксимація Rogers-Kwak, заснована на схемі Roe третього порядку точності. У моделях турбулентності для апроксимації конвективних складових застосовувалася схема TVD 3 обмежувачем потоків ISNAS третього порядку. Проведено порівняння результатів розрахунків обтікання циліндра 3 використанням моделі ламінарно-турбулентного переходу і без неї. Показано, що при низьких числах Рейнольдса, коли обтікання циліндра носить ламінарний характер, а слід турбулентний, використання моделі турбулентності Spalart-Allmaras призводить до розвитку турбулентного примежового шару на циліндрі i, як наслідок, до зміни положення точки відриву. Неправильне положення точки відриву впливас на розподіл тиску в донній частині циліндра і на інтегральні аеродинамічні характеристики. Застосування моделі переходу дозволяє адекватно відтворити ламінарний відрив поблизу передньої окрайки профілю з подальшим його приєднанням. Застосування однісї тільки моделі Spalart-Allmaras призводить до зайвої генерації турбулентної в'язкості. Показано, що застосування $\gamma$-Reө моделі ламінарно-турбулентного переходу якісно і кількісно покращус результати чисельного моделювання. Отримані результати чисельного моделювання обтікання кругового циліндра i аеродинамічного профілю NACA 4412 добре узгоджуються 3 експериментальними даними в широкому діапазоні чисел Рейнольдса.

Ключові слова: ламінарно-турбулентний перехід, аеродинамічний профіль, рівняння Нав'€Стокса, модель турбулентності.

A simulation of the flow around a cylinder and a NACA 4412 airfoil was carried out using the $\gamma$ $\gamma-\operatorname{Re}_{\theta}$ laminar-turbulent transition model and without it. Numerical simulation was performed on the basis of the Reynolds-averaged Navier-Stokes equations (URANS), using the Spalart-Allmaras differential one-parameter turbulence model. The system of initial equations was written with respect to an arbitrary curvilinear coordinate system. The coordination of the pressure and velocity fields was carried out using the method of artificial compressibility modified to calculate non-stationary problems. The integration of the system of initial equations was carried out numerically using the control volume method. For convective flows, a countercurrent Rogers-Kwak approximation was used based on the third-order Roe scheme. In turbulence models, the TVD scheme with a third-order ISNAS flow limiter was used to approximate convective terms. Comparison of the calculation results for a flow past a cylinder is carried out using the laminar-turbulent transition model and without it. It is shown that at low Reynolds numbers, when the flow around a cylinder is laminar, and the track isturbulent use of the Spalart-Allmaras turbulence model leads to the development of a turbulent boundary layer on the cylinder and, consequently, to a change in the position of the separation point. The incorrect position of the separation point affects the pressure distribution in the bottom of the cylinder and, as a result, the integral aerodynamic characteristics. The use of the transition model allows one to adequately reconstruct the laminar separation near the leading edge of the airfoil with its subsequent reattachment. The use of the Spalart-Allmaras model alone leads to excessive generation of turbulent viscosity. It is

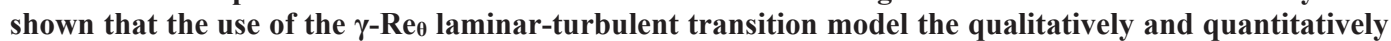
improves the results of numerical simulation. The results of numerical simulation of the flow around a circular cylinder and the NACA 4412 aerodynamic airfoil agree well with experimental data in a wide range of Reynolds numbers. model.

Key words: laminar-turbulent transition, aerodynamic airfoil, Navier-Stokes equations, turbulence

Введение. На сегодняшний день одной из проблем вычислительной аэродинамики является моделирование турбулентности и учет ламинарно-турбулентного перехода, а также обратного процесса - реламиниризации. Большое количество течений, встречающихся на практике, являются переходными. В этих течениях присутствует как ламинарная форма движения жидкости или газа, так и турбулентная. К таковым относятся течения в газовых турбинах, в теплообменниках ядерных реакторов, на крыльях летательных аппаратов (беспилотников) и др. Зачастую при рассмотрении такого рода задач ламинарной зоной пренебрегают и рассматривают всю область течения как турбулентную. От того, является ли течение ламинарным или турбулентным, зависит распределение трения, тепловых потоков и давления по поверхности обтекаемого тела. Пренебрежение зоной ламинарно-турбулентного перехода может привести к существенным погрешностям в определении интегральных 
характеристик. Полуэмпирические модели турбулентности, разработанные для замыкания осредненных по Рейнольдсу уравнений Навье-Стокса, не в состоянии смоделировать переход. Это привело к созданию нового направления в вычислительной аэродинамике, главной целью которого является моделирование ламинарно-турбулентного перехода.

Целью настоящей работы является изучение физических особенностей ламинарнотурбулентного перехода при обтекании кругового цилиндра и аэродинамического профиля NACA 4412.

Постановка задачи. Из всего многообразия моделей ламинарно-турбулентного перехода была выбрана наиболее успешная дифференциальная модель $\gamma$-Re $\theta$ Menter [3], базирующаяся на уравнении для коэффициента перемежаемости и уравнении для критического числа Рейнольдса потери импульса. Изначально данная модель разрабатывалась для применения совместно с моделью турбулентности $k$ - $\omega$ SST Menter [2]. В настоящей работе модель $\gamma$-Re $e_{\theta}$ ламинарно-турбулентного перехода была адаптирована для использования совместно с моделью турбулентности Spalart-Allmaras [5]. Математическое моделирование проведено с использованием нестационарных осредненных по Рейнольдсу уравнений Навье-Стокса (URANS), замкнутых дифференциальной однопараметрической моделью турбулентности Spalart-Allmaras [5]. В качестве начальных условий задавались параметры невозмущенного потока во всей расчетной области. На внешней границе применялись неотражающие граничные условия, для расчета которых использовался метод характеристик. На поверхности твердого тела ставилось условие прилипания. В модели турбулентности Spalart-Allmaras значение рабочей переменной на теле задавалось равным нулю, на выходной границе ставилось условие Неймана.

CFD пакет и его верификация. Для численного решения поставленной задачи использовался разработанный специализированный пакет вычислительной аэродинамики (CFD пакет) на основе уравнений Навье-Стокса, включая несколько дифференциальных моделей турбулентности, для расчета стационарных и нестационарных ламинарных и турбулентных течений. Система исходных уравнений записывалась относительно произвольной криволинейной системы координат. Согласование полей давления и скорости осуществлялось с помощью метода искусственной сжимаемости, модифицированного для расчета нестационарных задач [4]. Интегрирование системы исходных уравнений осуществлялось численно с использованием метода контрольного объема. Для конвективных потоков использовалась противопоточная аппроксимация Rogers-Kwak [4], основанная на схеме Roe третьего порядка точности. В модели турбулентности Spalart-Allmaras для аппроксимации конвективных слагаемых применялась схема TVD с ограничителем потоков ISNAS третьего порядка. Производные в вязких членах аппроксимировались центральноразностной схемой второго порядка. Алгоритм решения уравнений базируется на трехслойной неявной схеме с подитерациями по псевдовремени второго порядка точности по физическому времени. Полученная блочно-матричная система линейных алгебраических уравнений решалась методом минимизации обобщенной невязки GMRES c ILU(k) предобуславливанием.

Результаты и обсуждение. Преимущество использования модели перехода было продемонстрировано на задачах об обтекании кругового цилиндра в широком диапазоне чисел Рейнольдса и аэродинамического профиля NACA 4412.

Турбулентное обтекание цилиндра проводилось для пяти чисел Рейнольдса $10^{3}, 10^{4}, 10^{5}$, $10^{6}, 10^{7}$. Расчеты выполнены на сетке типа-О с количеством узлов $250 \times 300$. Для адекватного разрешения вязких эффектов в физической области вводится сильно неравномерная сетка со сгущением вблизи поверхности цилиндра и в следе. Первый шаг сетки от поверхности равен $10^{-6}$, количество точек в пограничном слое порядка 40 . Внешняя граница расположена на расстоянии 65 диаметров цилиндра, шаг интегрирования по времени $\Delta \mathrm{t}=0.005$. Проведено сравнение результатов расчетов обтекания цилиндра с использованием модели $\gamma-R_{\theta}$ ламинарно-турбулентного перехода и без нее. Обтекание цилиндра при числе Рейнольдса Re $=10^{3}$ характеризуется ламинарным режимом с последующей турбулизацией следа (рис. $1 \mathrm{a}$ ). При отсутствии модели перехода пограничный слой изначально является турбулентным (рис. 1 б). 

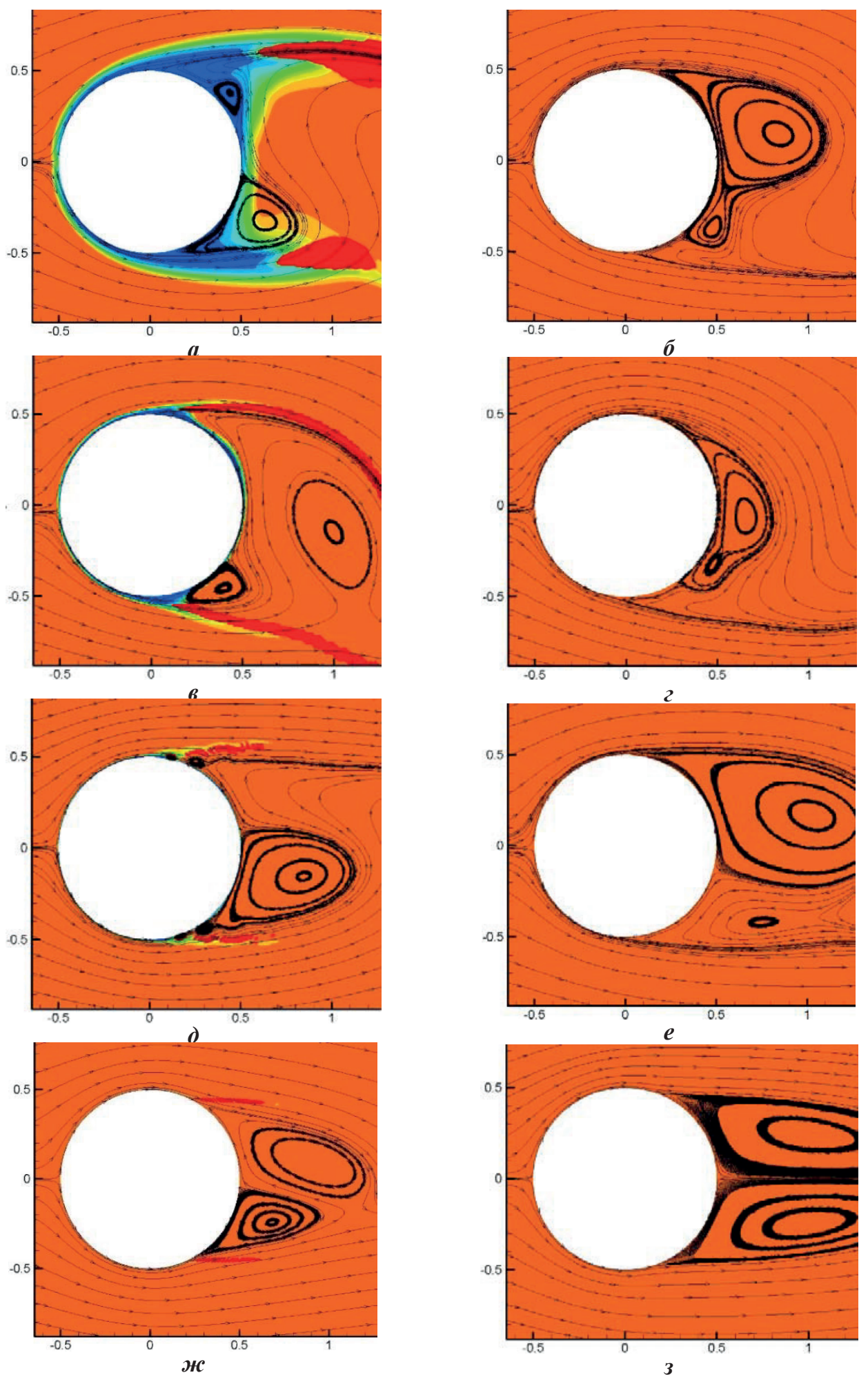

Рис. 1. Контуры перемежаемости и линии тока при обтекани цилиндра с моделью перехода

$\left(\mathrm{a}, \mathbf{в}\right.$, д, ж) и без нее $(б, \Gamma, \mathrm{e}, 3):\left(\mathrm{a}, \boldsymbol{\sigma}-\operatorname{Re}=10^{3} ; \mathrm{в}, \Gamma-\operatorname{Re}=10^{4} ;\right.$ д, $\left.\mathrm{e}-\operatorname{Re}=10^{5} ; ж, 3-\operatorname{Re}=10^{6}\right)$ 

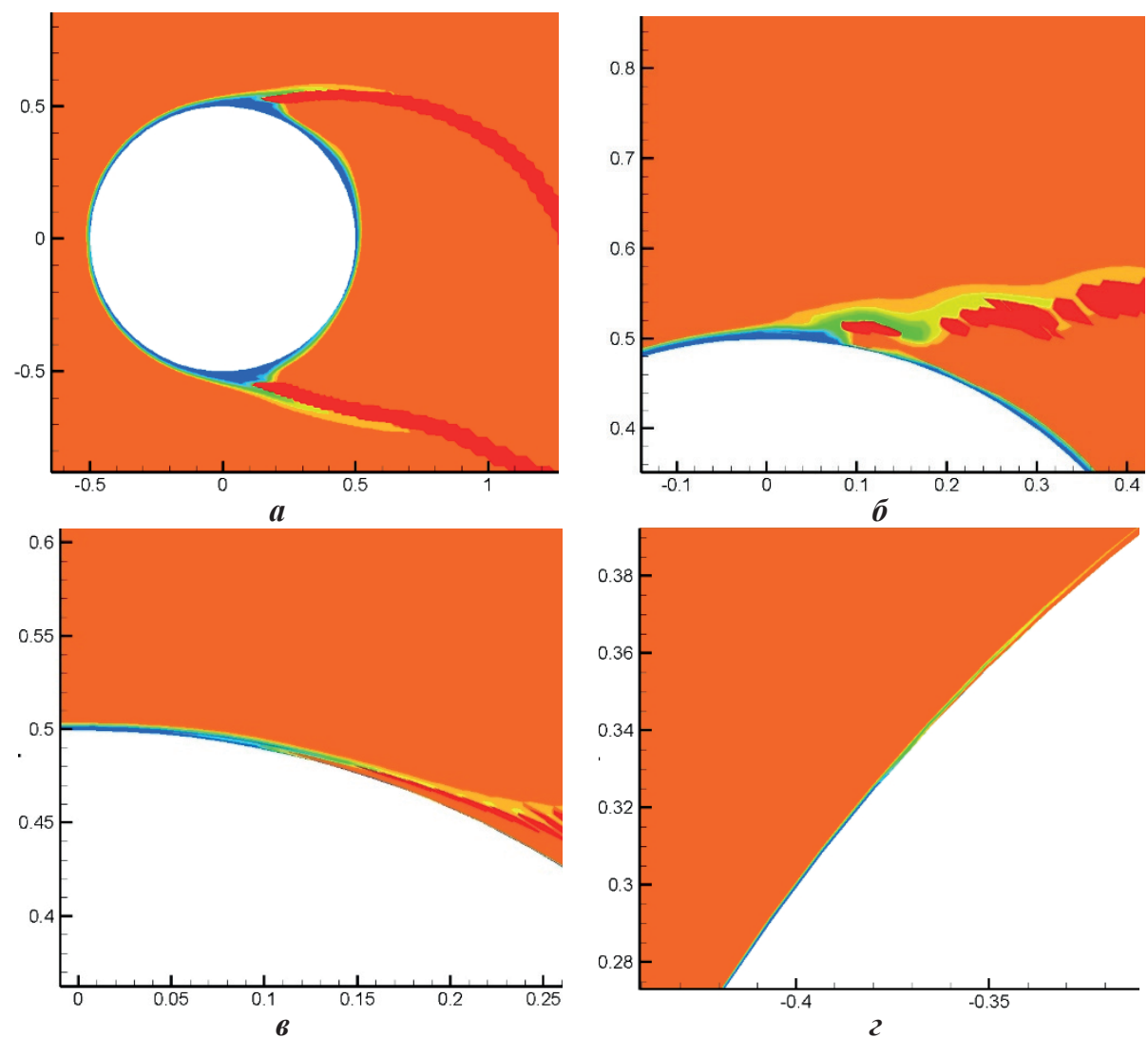

Рис. 2. Контуры перемежаемости при обтекании цилиндра $\left(\mathrm{a}-\operatorname{Re}=10^{4}, \sigma-\operatorname{Re}=10^{5}, \mathbf{B}-\operatorname{Re}=10^{6}, \Gamma-\operatorname{Re}=10^{7}\right)$

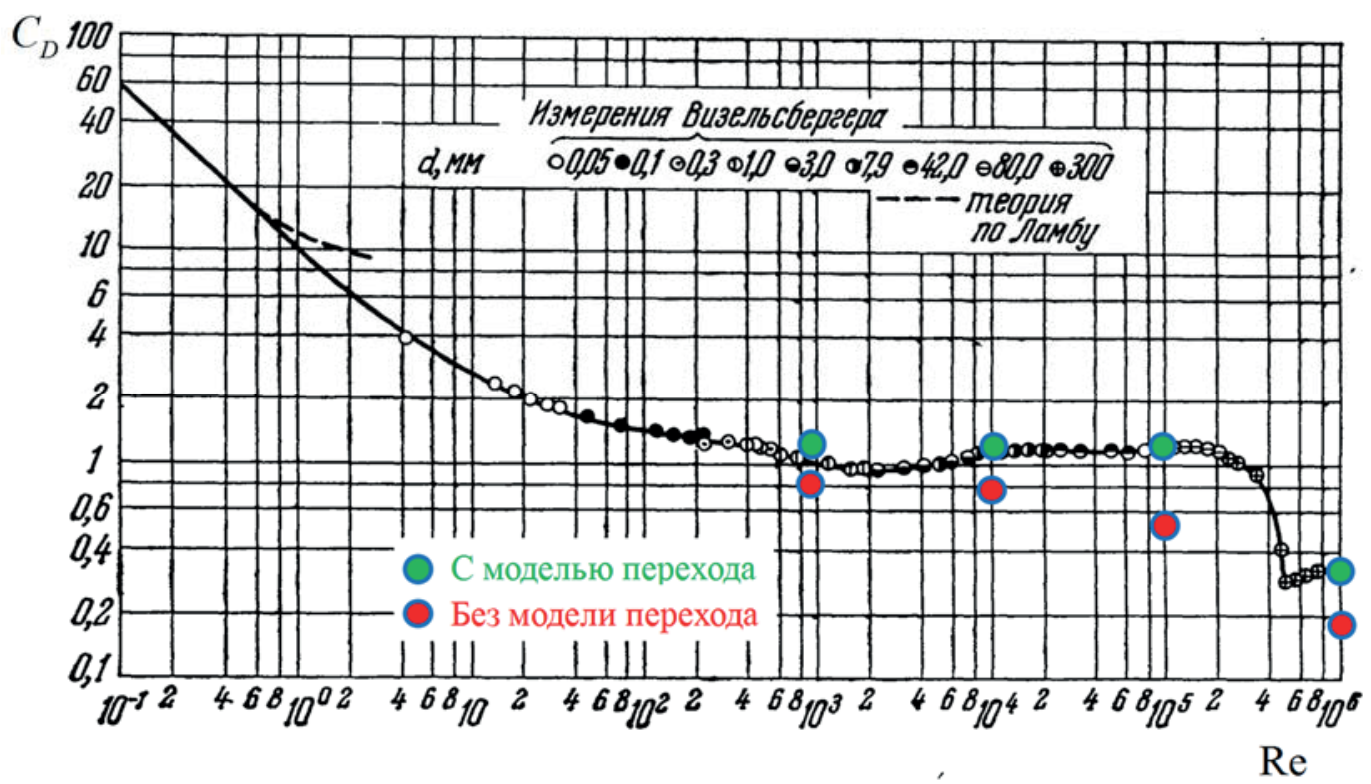

Рис. 3. Зависимость коэффициента сопротивления цилиндра от числа Рейнольдса [6] 

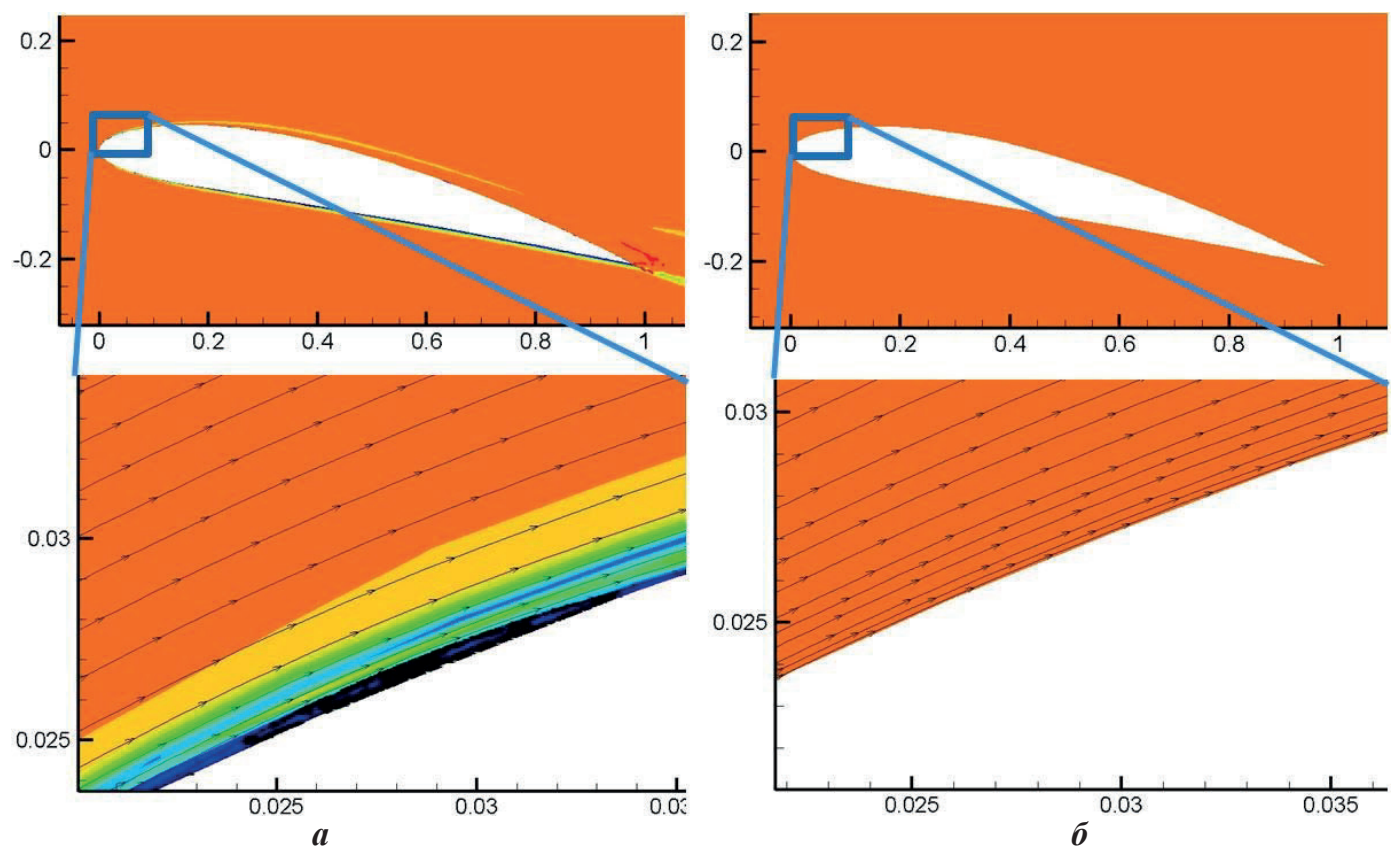

Рис. 4. Контуры перемежаемости и линии тока при обтекании профиля NACA 4412 $\left(\alpha=12^{\circ}\right)$ с моделью перехода (а) и без нее (б)

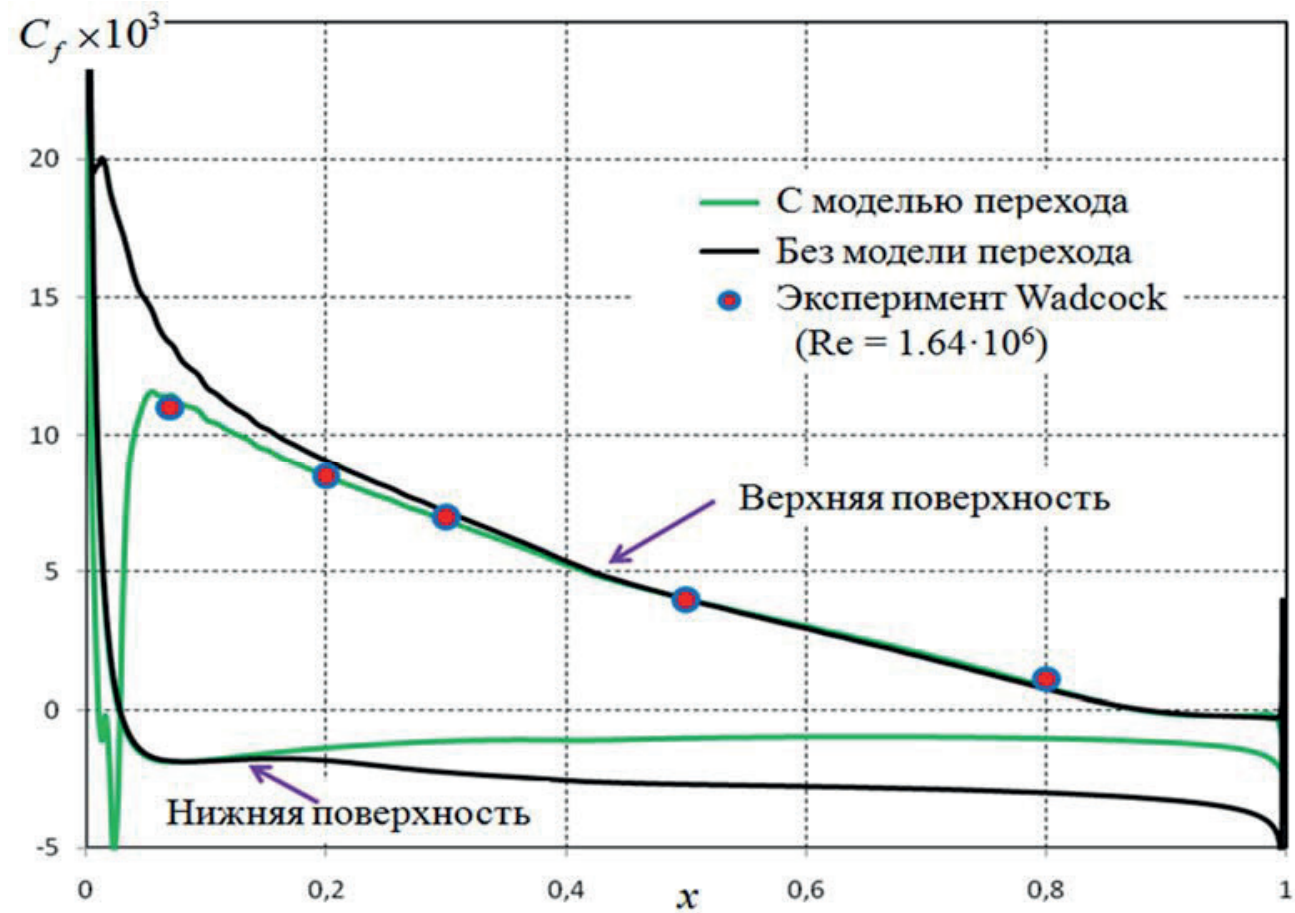

Рис. 5. Распределение коэффициента трения по поверхности профиля NACA $4412\left(\alpha=12^{\circ}\right)$

При числе Рейнольдса $\operatorname{Re}=10^{4}$ зона перемежаемости смещается ближе к поверхности цилиндра (рис. 1 в). Отрыв ламинарного пограничного слоя происходит при угле $85^{\circ} \div 90^{\circ}$. В 
то же время при моделировании обтекания цилиндра без модели ламинарно-турбулентного перехода турбулентный пограничный слой отрывается гораздо позже (рис. 1 г).

Ламинарный пограничный слой при числе Рейнольдса $10^{5}$ отрывается при угле приблизительно $90^{\circ}$ с последующей его турбулизацией (рис. 1 д). В этих трех случаях при числах Рейнольдса $10^{3}, 10^{4}, 10^{5}$ имеет место отрывной переход.

При числе Рейнольдса $\mathrm{Re}=10^{6}$ ламинарно-турбулентный переход происходит на поверхности цилиндра (рис. 1 ж). Отрывается уже турбулентный пограничный слой. Для случая, когда не используется модель перехода, обтекание цилиндра носит стационарный характер из-за излишней генерации турбулентной вязкости (рис. 1 з).

На рис. 2 представлено изменение зоны перемежаемости и характера ламинарнотурбулентного перехода от числа Рейнольдса. С увеличением числа Рейнольдса зона ламинарно-турбулентного перехода смещается вверх по потоку и меняется характер перехода от отрывного к естественному.

Применение модели ламинарно-турбулентного перехода качественно и количественно улучшает результаты численного моделирования. Показано, что при низких числах Рейнольдса, когда обтекание цилиндра носит ламинарный характер, а след турбулентный, использование модели турбулентности SA приводит к развитию турбулентного пограничного слоя на цилиндре и, как следствие, к изменению положения точки отрыва. Неправильное положение точки отрыва оказывает влияние на распределение давления в донной части цилиндра и на интегральные аэродинамические характеристики (рис. 3). Полученные результаты численного моделирования обтекания кругового цилиндра в широком диапазоне чисел Рейнольдса хорошо согласуются с экспериментальными данными [1].

Еще одной задачей, на которой проводилось тестирование модели $\gamma$ - $R e_{\theta}$ ламинарнотурбулентного перехода, было обтекание аэродинамического профиля NACA 4412. Ниже представлены результаты расчетов, проведенные для неподвижного профиля NACA 4412, применительно к экспериментальным данным из работы Wadcock [6] при числе Рейнольдса $1.64 \cdot 10^{6}$, определенном по хорде профиля, скорости невозмущенного потока $29.1 \mathrm{~m} / \mathrm{c}$. Уровень турбулентности набегающего потока равен $0.0087 \%$.

Расчеты проведены на сетке типа-О с общим числом узлов $1.2 \cdot 10^{5}$. В направлении по нормали к поверхности в пограничном слое находилось порядка 150 точек. Первый шаг сетки от поверхности профиля равен $3 \cdot 10^{-6}$, что обеспечивает максимальное значение $\mathrm{y}^{+}<0.1$.

При турбулентном обтекании аэродинамического профиля NACA 4412 при угле атаки $12^{\circ}$ на подветренной части передней кромки формируется ламинарный отрыв (рис. 4 а). В точке присоединения потока происходит резкая турбулизация пограничного слоя, и дальнейшее обтекание носит присоединенный характер, за исключением небольшой отрывной зоны вблизи задней кромки. К тому же обтекание наветренной стороны профиля носит полностью ламинарный характер. Применение модели турбулентности SpalartAllmaras без учета ламинарно-турбулентного перехода приводит к излишней генерации турбулентной вязкости и не дает возможности адекватно описать эти процессы (рис. 4 б). В результате весь пограничный слой на профиле является турбулентным и без ламинарного отрыва вблизи передней кромки. Применение дифференциальной модели $\gamma-R_{\theta}$ позволяет учесть эти сложные явления и адекватно описать ламинарно-турбулентный переход на поверхности профиля (рис. 5). Эти различия наглядно демонстрирует распределение коэффициента трения по поверхности профиля, где виден отрыв. Полученные результаты хорошо согласуются с экспериментальными данными Wadcock [6].

Заключение. Преимущество использования $\gamma$ - $\operatorname{Re}_{\theta}$ модели перехода было продемонстрировано на задачах об обтекании кругового цилиндра в широком диапазоне чисел Рейнольдса и аэродинамического профиля NACA 4412. Проведено сравнение результатов расчетов обтекания цилиндра с использованием модели $\gamma-R_{\theta}$ ламинарнотурбулентного перехода и без нее. Показано, что при низких числах Рейнольдса, когда обтекание цилиндра носит ламинарный характер, а след турбулентный, использование модели турбулентности Spalart-Allmaras приводит к развитию турбулентного пограничного слоя на цилиндре и, как следствие, к изменению положения точки отрыва. Неправильное 
положение точки отрыва оказывает влияние на распределение давления в донной части цилиндра и на интегральные аэродинамические характеристики. Применение модели перехода позволяет адекватно воспроизвести ламинарный отрыв вблизи передней кромки профиля с последующим его присоединением. Применение одной только модели SpalartAllmaras приводит к излишней генерации турбулентной вязкости. Показано, что применение $\gamma-R e_{\theta}$ модели ламинарно-турбулентного перехода качественно и количественно улучшает результаты численного моделирования. Полученные результаты численного моделирования обтекания кругового цилиндра и аэродинамического профиля NACA 4412 хорошо согласуются с экспериментальными данными в широком диапазоне чисел Рейнольдса.

\section{Библиографические ссылки}

1. Шлихтинг, Г. Теория пограничного слоя [Текст] / Г. Шлихтинг. - М.: Наука, 1974. $-712 \mathrm{c}$.

2. Menter, F. R. Transition Modelling for General Purpose CFD Codes [Текст] / F. R. Menter, R. B. Langtry, S. Völker // Journal Numerical Mathematics. - 2006. - Vol. 4. - P. $277-$ 303.

3. Menter, F. R. Zonal Two Equation k- $\omega$ Turbulence Models for Aerodynamic Flows [Текст] / F. R. Menter // Journal Numerical Mathematics. - 1993. - Vol. 2. - P. 117- 124.

4. Rogers, S. An upwind differencing scheme for the incompressible Navier-Stokes equations [Текст] / S. Rogers, D. Kwak // Journal Numerical Mathematics. - 1991. - Vol. 8. - P. 43 - 64.

5. Spalart, P. R. A one-equation turbulence model for aerodynamic flow [Текст] / P. R. Spalart, S. R. Allmaras // AIAA Paper. - 1992. - Vol. 12, № 1. - P. 439-478.

6. Wadcock, A. J. Investigation of low-speed turbulent separated flow around airfoils / A. J. Wadcock // NASA-CR-177450. - 1987. - P. 66.

Надійшла до редколегї 18.10.2019

Anatoly P. Lukisha

Institute of Geotechnical Mechanics named by N.S. Poljakov of the National Academy of Sciences of Ukraine

The article is devoted to the development of a method for recalculating the thermal-hydraulic characteristics of direct-flow cylindrical steam-generating channels operating on a Freon coolant from the boundary conditions of the second kind for the boundary conditions of the first kind. The need for the development of such recalculation procedure is due to the presence in the literature of calculated dependences describing the heat transfer during evaporation of the coolant in porous channel for boundary conditions of the second kind, while the practical plan problems are often conditioned by other boundary conditions, in particular boundary conditions of the first kind.

(C) Anatoly P. Lukisha, 2019 